\title{
Dual-layer flexible THz metamaterial realization of electromagnetically induced transparency and absorption
}

\author{
Yonggang Zhang ${ }^{1}$ \\ ${ }^{1}$ School of Electrical and Information Engineering, Anhui University of Science and Technology, \\ Huainan 232001, China; \\ aygzhang2000@163.com
}

Keywords-flexible, THz metamaterial, electromagnetically induced transparency. Abstract - Terahertz electromagnetically induced transparency (EIT) metamaterial based on the dual layered resonator array was fabricated on flexible polyimide films using photolithography technique. The samples were measured by terahertz time-domain spectroscopy (THz-TDS). The reflectance and absorption of the samples were also extracted. The fabricated flexible samples have the characteristics of electromagnetically induced transparency (EIA).

\section{Introduction}

Electromagnetically induced transparency (EIT) is a kind of quantum interference effect, which was first observed in three level atomic system [1,2]. The classic system can also be used to realize EIT. The research of using metamaterial mimicking EIT is one of the hot issues in recent years [3,4]. The classical analog were presented in the literatures [4-6]. When the metamaterial can be seen as a single layer structure, its effective response can be described by an electric current sheet with equivalent parameter of surface conductivity $\sigma$ [6-8]. The scattering parameters of an electric current sheet can be calculated from the parameter $\sigma$.

Rapidly expanding $\mathrm{THz}$ technology has various potential application in many fields. Polyimide is one of flexible substrates with low loss, transparent at $\mathrm{THz}$ band. It can make the device more flexible and convenient attached on the surface of different shapes.

\section{Sample design, simulation, experimental results, discussion}

In this paper, a dual layer flexible EIT metamaterial was fabricated using microfabriaction process. First, a 5- $\mu \mathrm{m}$ thick polyimide film was spin coated onto the silicon wafer and soft baked. Second, 200-nm-thick gold layer was deposited on the polyimide film using thermal evaporator and patterned by lift off technique to form bottom metallic layer of radiative resonator array. Third, a $10-\mu \mathrm{m}$-thick polyimide film was spin coated and soft baked. A metallic layer of dark resonator array was subsequently formed onto the polyimide layer using thermal evaporation and lift-off technique. The schematic representation of our structure is shown in Fig. 1. 


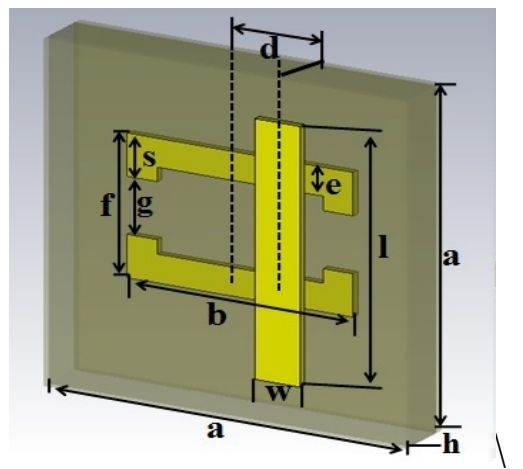

Fig. 1. Schematic representation of our structure. The dimensions of the sample is $a=220 \mu \mathrm{m}$. Feature sizes are $b=150 \mu \mathrm{m}, e=20 \mu \mathrm{m}, f=100 \mu \mathrm{m}, g=40 \mu \mathrm{m}, h=10 \mu \mathrm{m}, l=180 \mu \mathrm{m}, w=30 \mu \mathrm{m}$, $s=30 \mu \mathrm{m}$, The translational offset between the centers of dark radiative resonator is $d$. The incident wave is polarized along the $y$ axis.

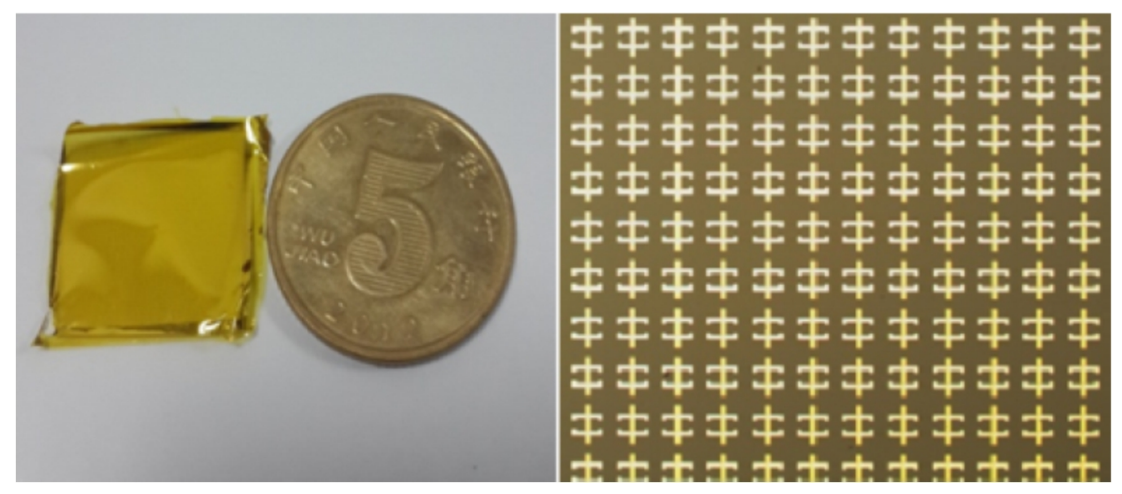

Fig. 2. Optical images for the EIT matematerial

Using alignment mark, the different translational offset $d$ between center of resonants were fabricated. Then, the top polyimide layer with a thickness of $5 \mu \mathrm{m}$ was spin coated. Finally, the whole structure was peeled off from the silicon wafer.

Optical images for the fabricated EIT matematerial are shown in Fig. 2. All samples were measured using terahertz timedomain spectroscopy (THz-TDS). Transmission spectrum of the all samples are shown in Fig. 3(a). When $d$ increased, the transparency window which is a typical characteristic of EIT appears. With the increasing of $d$, the coupling effect becomes stronger, as a result, the transmittance peak gradually increases.

The $\mathrm{THz}$ transmission spectra of each sample were simulated using the finite integral time domain simulation software. The simulation shown in Fig. 3(b) are consistent with experimental results. The relationship between the surface conductivity and scattering parameters were discribed in [6]. The scattering parameters of the flexible EIT metamaterial can be calculated through the experimental transmission. Fig. 4(a) is reflectance calculated and the Fig. 4(b) is absorption obtained. It can be seen that with the increasing of offset $d$, the electromagnetically induced absorption (EIA) characteristics become obvious. This shows that the flexible metamaterials we fabricated not only implement EIT, but also has the characteristics of EIA. 

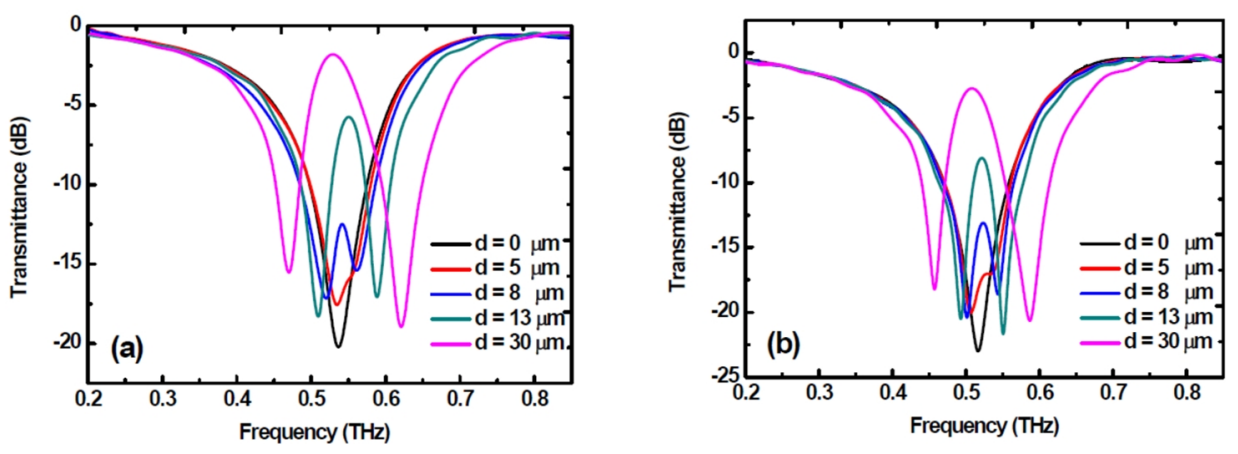

Fig. 3: Transmission spectrum of flexible EIT metamaterials with different $d$. (a): experiment; (b): simulation.
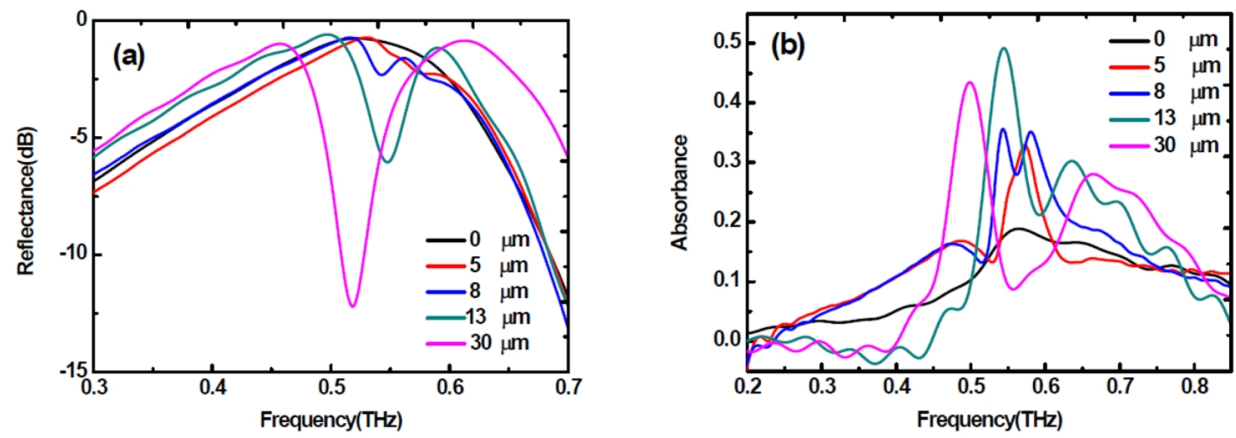

Fig. 4. Reflectance and absorbance calculated from experimental data, (a): reflectance; (b): absorbance.

\section{Conclusions}

In conclusion, we demonstrated dual layer $\mathrm{THz}$ metamaterials built on highly flexible polyimide substrates. Through tuning the symmetry of two coupled resonators onto two polyimide layers, the transition of spectral response from single resonance to EIT were observed both in numerical simulation and experimental results. Furthermore, the absorption characteristics of these samples were comprehensively studied. These results pave the way for developing multilayered and flexible THz optical devices.

\section{Acknowledgments}

This work is supported by the Key Programs of Natural Science Foundation of Higher Education Institution of Anhui Province, China (KJ2016A195); Programs of Doctor Foundation of Anhui University of Science and Technology (11679).

\section{References}

[1] S. E. Harris, "Electromagnetically induced transparency," Physics Today vol.50, 36-42 (1997)

[2] M. Fleischhauer, A. Imamoglu, and J. P. Marangos, "Electromagnetically induced transparency: Optics in coherent media," Review of Modern Physics 77, 633-673, (2005)

[3] S. Zhang, D. A. Genov, Y. Wang, M. Liu, and X. Zhang, "Plasmon-induced transparency in metamaterials," Physical Review Letters 101, 047401, (2008) 
[4] N. Liu, L. Langguth, T.Weiss, J. Kästel, M.Fleischhauer, T. Pfau, and H.Giessen, "Plasmonic analogue of electromagnetically induced transparency at the Drude damping limit," Nature Materials 8, 758-762, (2009)

[5] C. L. G. Alzar, M. A. G. Martinez, P. Nussenzveig. "Classical analog of electromagnetically induced transparency," American Journal of Physics, 70: 37, (2002)

[6] P. Tassin, L. Zhang, R. Zhao, et al. "Electromagnetically Induced Transparency and Absorption in Metamaterials: The Radiating Two-Oscillator Model and Its Experimental Confirmation," Physical review letters, 109(18): 187401, (2012)

[7] P. Tassin, T. Koschny, and C. M. Soukoulis, "Effective material parameter retrieval for thin sheets Theory and application to graphene, thin silver films, and single-layer metamaterials," Physica (Amsterdam) 407B, 4062, (2012)

[8] Y. G. Zhang, J. B. Wu, et al, Tailoring electromagnetically induced transparency effect of terahertz metamaterials on ultrathin substrate. Science China (Information Sciences). 59, 042414,(2016) 